\title{
A CLINICAL APPRAISAL OF THYMIC LESIONS AND ANTERIOR MEDIASTINAL LYMPHOMATA
}

\author{
BY \\ B. T. LE ROUX \\ From the Department of Thoracic Surgery, University of Edinburgh
}

(RECEIVED For PUBLICATION NOVEMBER 11, 1960)

Surgical experience of thymic lesions is usually acquired by investigating patients who present in one of three ways: with myasthenia gravis, with a symptomless opacity found at routine radiography; or with evidence of a malignant mediastinal tumour, which often includes obstruction of the superior vena cava. The function of the thymus is not known, and the histological interpretation of its normal variations and of its pathology is difficult and confusing (Seybold, McDonald, Clagett, and Good, 1950 ; Fisher, Coburn, and Effler, 1952 ; Effler and McCormack, 1956). In adults, cysts and tumours in the anterior mediastinum, where this term is used to include the anterior part of the superior mediastinum, inevitably lie close to the thymic remnant, and when such lesions are resected inclusion of thymus in an operative specimen does not necessarily mean that the lesion resected had originated in the thymus (Seybold et al., 1950). The disorders of the reticulo-endothelial system involve the thymus because of the high concentration of lymphocytes in this organ. The reticuloses or malignant lymphomata will frequently affect the thymus and some of them may originate in it. A malignant lymphoma confined to the anterior mediastinum, while it may have originated in the thymus, may also have originated in mediastinal lymph glands and have invaded the thymus, or it may have had a local but multicentric origin. A malignant thymoma, if it is not a carcinoma, is nearly always a malignant lymphoma (Effler and McCormack, 1956). It is not always possible to establish that a carcinoma is unequivocally of thymic origin ; the reported cases of thymic carcinoma have rarely been confined to the thymus, and many have probably arisen in lung (Blades, 1941; Willis, 1948) or from a mediastinal teratoma with a malignant epithelial component (Ringertz and Lidholm, 1956).

A variety of thymic lesions have been seen over the past 12 years in the Regional Thoracic Unit in Edinburgh. These cases are presented from the standpoint of their clinical features and the operative findings in those submitted to thoracotomy, rather than from that of their histological features, because there is little ambiguity in the clinical assessment and the surgical aspects of these cases, whereas there is little agreement on their histological interpretation. In some instances, where operative material was examined by a panel of pathologists, as many as seven different interpretations of the histological findings were offered. A histological report is an expression of an opinion that must be interpreted in conjunction with clinical, radiographic, and operative information, and such an opinion is in no sense oracular or beyond dispute.

Two examples of heterotopic thymic tissue have been encountered, and in one the lesion was resected. Four cysts, said to be of thymic origin, have been resected; in one there was a large solid element indisputably thymic. Seven solid, wellencapsulated, and apparently benign tumours have been enucleated from the anterior mediastinum; the histological interpretation of these tumours has varied from thymic hyperplasia to benign or possibly malignant thymoma. In six patients solid tumours, but locally invasive, have been resected from the anterior mediastinum; four of these patients were symptomless; histologically the six tumours were called malignant, probably or possibly of thymic origin. In this series, therefore, six out of 13 solid tumours of the thymus were invasive in comparison with a $25 \%$ incidence of invasive tumours in a series reported by Seybold et al. (1950), the absence of invasive tumours in a group of 19 cases reported by Ringertz and Lidholm (1956), and an incidence of eight invasive tumours in a series of 19 reported by Larmi (1960).

During the same period 19 patients with malignant lymphomata, 10 of them with superior caval obstruction, have been encountered. These patients all had an anterior mediastinal opacity, 
and at the time when they were first seen the only clinical evidence of dissemination of the lesion beyond the mediastinum was cervical lymphglandular enlargement in some. It is probable that these malignant lymphomata originated in the anterior mediastinum, and it is possible that the lymphomata may have originated in the thymus.

During the same 12 years thymectomy was undertaken in 18 patients who had myasthenia gravis. No patient with myasthenia gravis undergoing thymectomy had a thymic tumour; in the myasthenics the resected thymus was reported to be histologically normal, although Simpson (1960) disputes the normality of the thymus in these cases. No patient who had a thymic tumour had or has, so far, developed myasthenia gravis.

This experience of the absence of a relationship between thymic tumours and myasthenia is different from that of Eaton, Clagett, Good, and McDonald (1949), who found that $15 \%$ of patients with myasthenia gravis had thymic tumours; of Ringertz and Lidholm (1956), who found that $25 \%$ of patients with thymic tumours had myasthenia gravis ; and of Seybold et al. (1950), who found that $75 \%$ of patients with a "thymoma" had myasthenia gravis. These last authors defined the term thymoma and described the features of such a tumour. Malignant thymic tumours in myasthenia gravis have also been described (Clagett and Eaton, 1943; Murray and McDonald, 1945 ; Keynes, 1946 and 1955 ; Reid and Marcus, 1949 ; Effler and McCormack, 1956). Most authors fail to recognize histological criteria by which thymic tumours associated with myasthenia gravis can be distinguished from those unaccompanied by myasthenia (Fisher et al., 1952 ; Larmi, 1960). Andritsakis and Sommers (1959) suggested criteria for the recognition not only of thymic tumours associated with myasthenia gravis but also of those thymic lesions associated with Cushing's syndrome and with refractory anaemia. These authors suggested, on the basis of 50 cases, a pathological classification which they believed should supersede what they refer to as the "primitive inclusive term, thymoma." Their classification included 13 different types of thymic lesion, 12 of which may be either benign or malignant.

\section{HeTERotopic ThyMUS}

The epithelial elements of the thymus develop mainly and possibly exclusively from the endodermal outgrowths of the third branchial (pharyngeal) pouches. Early in the third month of foetal development the primordium of the thymus is a bilobed mass of small epithelial cells that is later invaded by lymphocytic elements. As the heart and great vessels descend, the consequence mainly of growth of the neck and the unfolding of the embryo, the thymus adopts its anatomical position in the mediastinum, immediately deep to the manubrium sterni, its inferior pole extending on to the pericardium. Just as thyroid tissue, by remaining adherent to the aortic sac, may develop in heterotopic sites in the mediastinum (le Roux, 1961), so may the thymus, by retaining its close association with the great vessels, develop in ectopic sites in the mediastinum. One example of such an aberrant thymus is described below. Examples of thymic hyperplasia or of thymomata developing at the pulmonary hilum have also been reported by Alter and Osnato (1930), Hardymon and Bradshaw (1944), and by Ringertz and Lidholm (1956). Intrapulmonary ectopic thymus has been recorded (McBurney, Clagett, and McDonald, 1951).

Aberrant thymus and thyroid have been described in the trachea and larynx. Cooper (1950) described a case of thyroid tissue in the trachea separated by three tracheal rings from the parent organ which ultimately caused death from asphyxia ; and Willis (1958) describes a case originally reported by Wadon in 1934 of thymus in the trachea. An example of asphyxial death in an infant, the consequence of obstruction of the airway from heterotopic thymus in the larynx, is also described below.

A healthy, symptomless 13-year-old girl was radiographed during a mass radiography campaign. A well-defined spherical opacity was demonstrated at the left border of the heart, lying in the lateral film immediately below the pulmonary hilum (Fig. 1). Preliminary investigations were unhelpful. At left thoracotomy in 1957 a solid tumour $3 \mathrm{~cm}$. in diameter was found in the oblique fissure of the lung, covered by visceral pleura reflected from the upper and lower lobes. The stem artery and its lingular and anterior basal branches were stretched over the surface of the tumour. The pleural reflection was incised, the branches of the pulmonary artery were displaced, and the tumour was shelled cleanly from its bed. A small bronchial artery required ligation. Recovery from thoracotomy was uneventful and the girl remains well. The anterior mediastinum was not examined with the specific intention of identifying the thymus. The microscopic features of the resected specimen were those of thymus with lymphoid follicles in a slightly greater proportion and epithelial elements in a somewhat smaller proportion than is usual.

In 1952 a baby girl was admitted during the fourth week of life with the clinical features of respiratory 


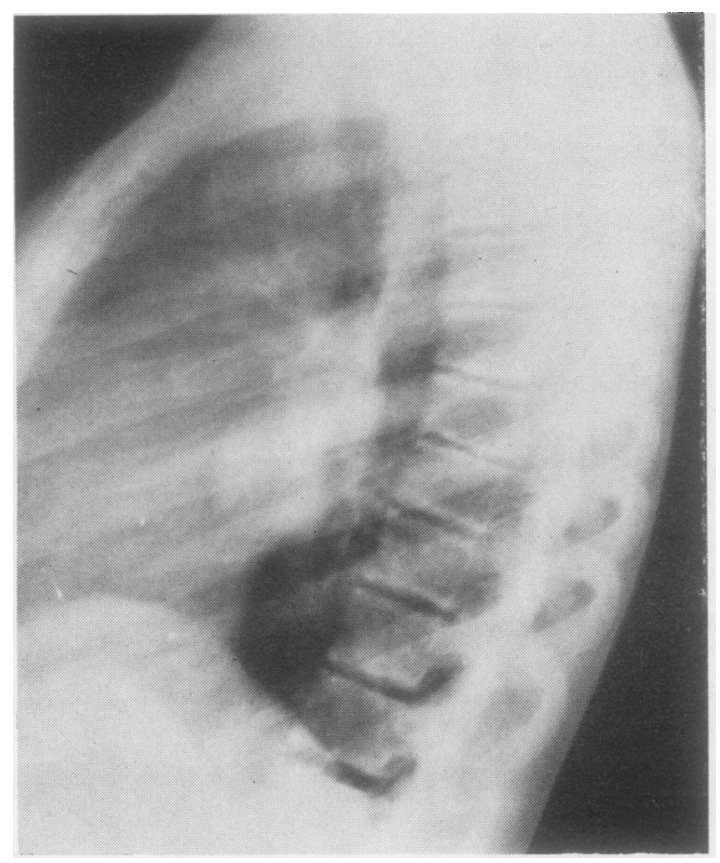

FIG. 1.-Heterotopic thymus in the oblique fissure of the left lung.

obstruction and with a normal chest radiograph. The child was acutely distressed; its airway was examined through a Negus suckling bronchoscope and no abnormality was found. The child's condition varied over several weeks; repeated bronchoscopies both diagnostic and therapeutic did not demonstrate any abnormality; a tracheostomy was made and retained for a month during which time the child continued to have less severe episodes of acute respiratory obstruction. After three months in hospital she remained well enough over a sufficiently long period for the mother to be encouraged to take her home; she was readmitted in urgent respiratory distress a few days later. During a subsequent episode of acute respiratory obstruction cardiac arrest occurred; the heart was re-started without the need for direct massage. At 8 months the child died during yet another episode of respiratory obstruction. The trachea and bronchi had been carefully examined many times, and the larynx had been scrutinized by experts; no lesion had been found. At necropsy there was a tumour in the larynx immediately below the left vocal cord. The tumour was covered by normal mucosa, and it had the histological features of normal thymus. There was a normal complement of thymic tissue in the anterior mediastinum without connexion with the laryngeal thymus.

\section{Thymic Cysts}

Four patients have been submitted to thoracotomy because of opacities in the anterior mediastinum. In each a cyst was found which was subsequenly shown histologically to be thymic or $\frac{\mathrm{C}}{0}$ to have thymic tissue so intimately incorporated $\bar{\omega}$ in its walls that no other origin could reasonably $\overrightarrow{\widehat{D}}$ be adduced. None of these four patients had symptoms. All were referred because of abnormal के routine radiographs. One was a woman of 54 , one $\overrightarrow{0}$ a boy of 18, and two were men of 67 and 71 years $\vec{\overrightarrow{ }}$ of age respectively. Investigations, including $\omega$ angiography in the woman to exclude aneurysm, did not help pre-operative diagnosis. Radiographs $\vec{x}$ of three of these four patients are shown (Figs. $\vec{\sigma}$ 2-7). In three a right thoracotomy was made and iv one was approached from the left. Recovery from $\vec{\circ}$ resection of the mediastinal cyst was uneventful $\circ$ in all, and all are well eight years to eight months $\frac{\mathrm{S}}{\mathrm{C}}$ after thoracotomy. In the last of these four $\vec{c}$ patients (Fig. 6) a provisional diagnosis of thymic cyst was made on the basis of the pre- $\Phi$ operative radiographs because of the similarity $\overrightarrow{\mathscr{E}}$ between the films and those of the boy of 18 (Figs. 4 and 5).

The lesion approached from the left was confined to the left hemithorax and lay in the anterior mediastinum. Incision of the mediastinal pleura reflected over the cyst allowed enucleation without dividing a pedicle. The cyst measured 5 $\mathrm{cm}$. in diameter, and was described by a panel of pathologists as of thymic origin and lined by ciliated columnar epithelium.

In the 54-year-old woman (Figs. 2 and 3) a thinwalled translucent multilocular cyst was found in the anterior mediastinum, extending as far to the left as it did to the right. There was no pedicle; the cyst extended as high as the root of the neck, and caudally over the aorta and pericardium. Histologically the wall was lined by columnar epithelium. in places ciliated, set on a delicate stroma of fibrous tissue with small collections of lymphocytes and Hassall's corpuscles scattered throughout its wall.

The similarity between the radiographs of the $N$ boy of 18 and the man of 71 has already been $N$ mentioned (Figs. 4-6). The boy was seen in 1952 and the man in 1960 . The operative findings in both were similar: a thin-walled translucent multilocular cyst extended from the diaphragm to the root of the neck; in the man the cyst was

FIGS. 2 and 3.-A thymic cyst in the anterior mediastinum. The recognition of an opacity in the postero-anterior view is difficult. but the patient was nevertheless referred for investigation becau se a mass radiograph was recognized as abnormal. The opacity above the pericardium in the anterior mediastinum is more clearly seen in the lateral view.

FIGS. 4 and 5.-A thymic cyst extending from the root of the neck to the diaphragm. 

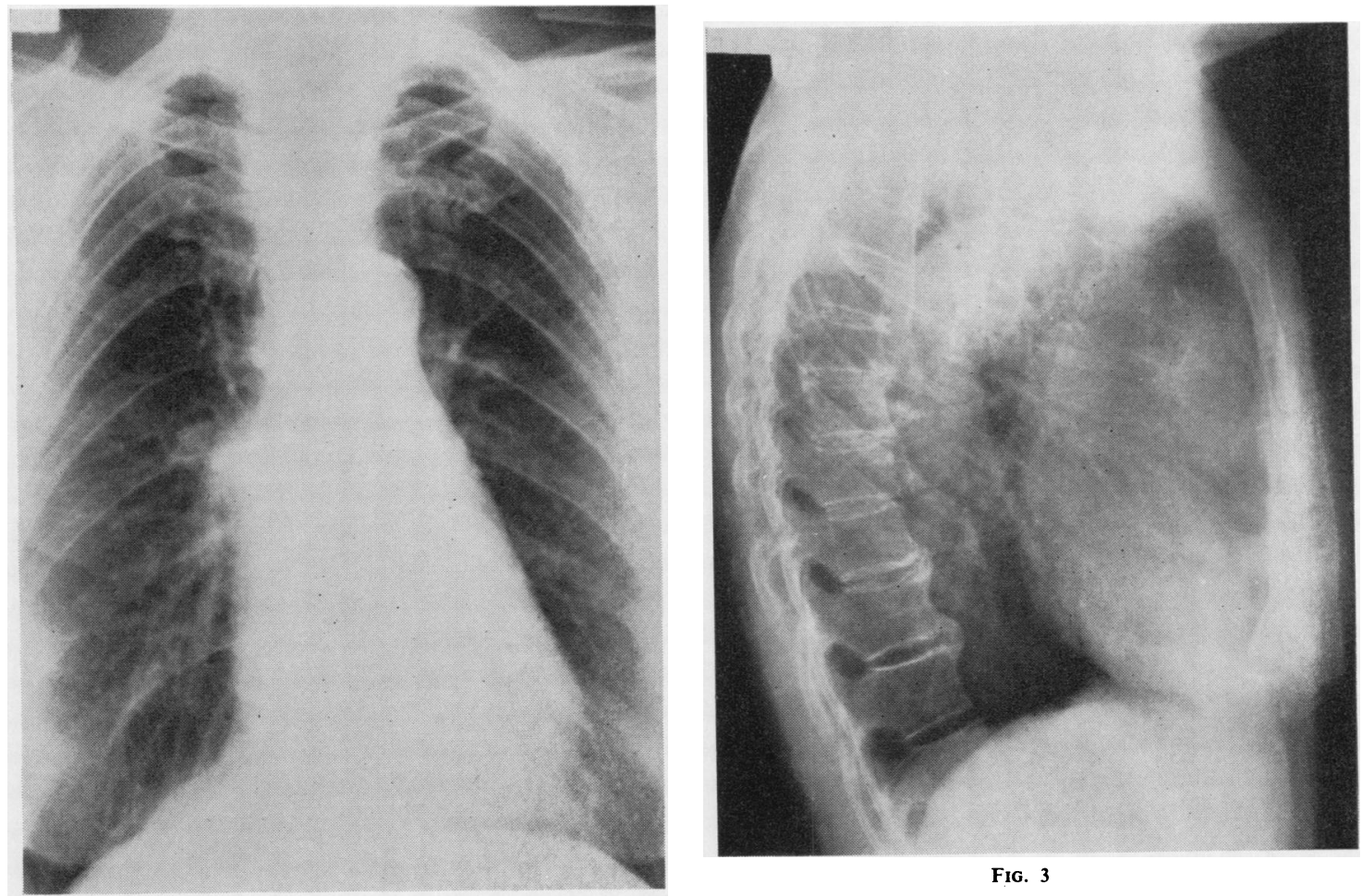

Fig. 2

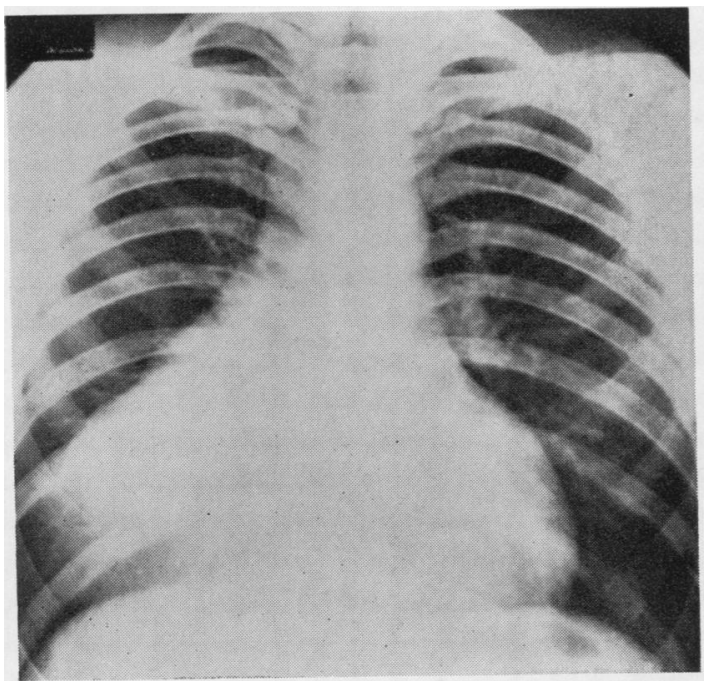

Fig. 4

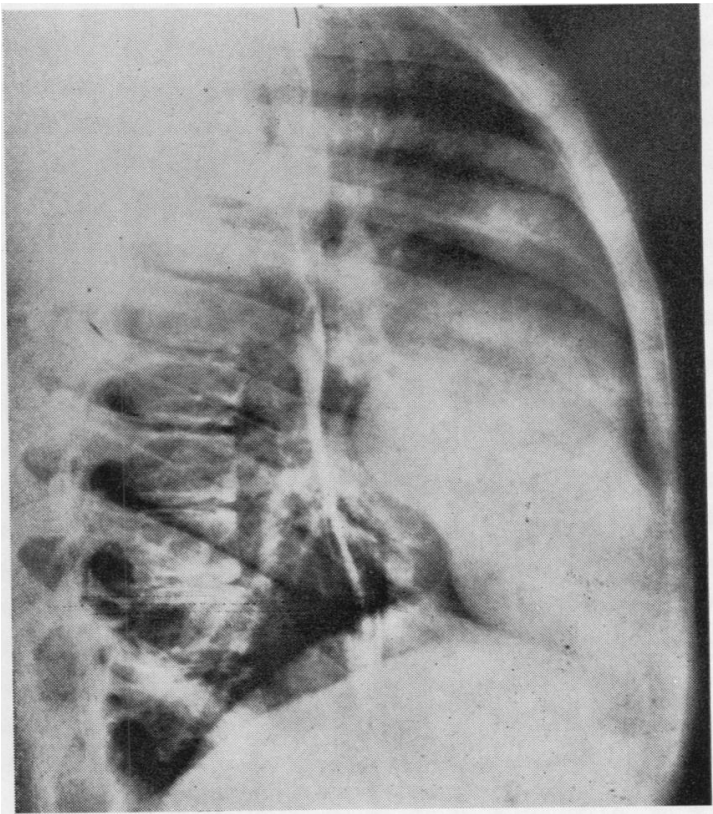

F10. 5 


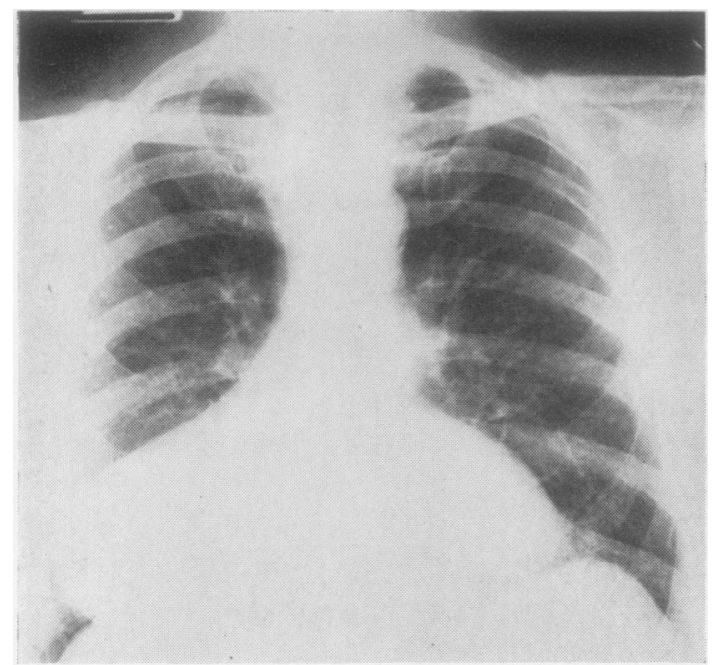

FIG. 6.-This radiograph is so similar to that illustrated in Fig. 4 that the diagnosis of thymic cyst was suggested pre-operatively. Histological verification of this diagnosis was obtained.

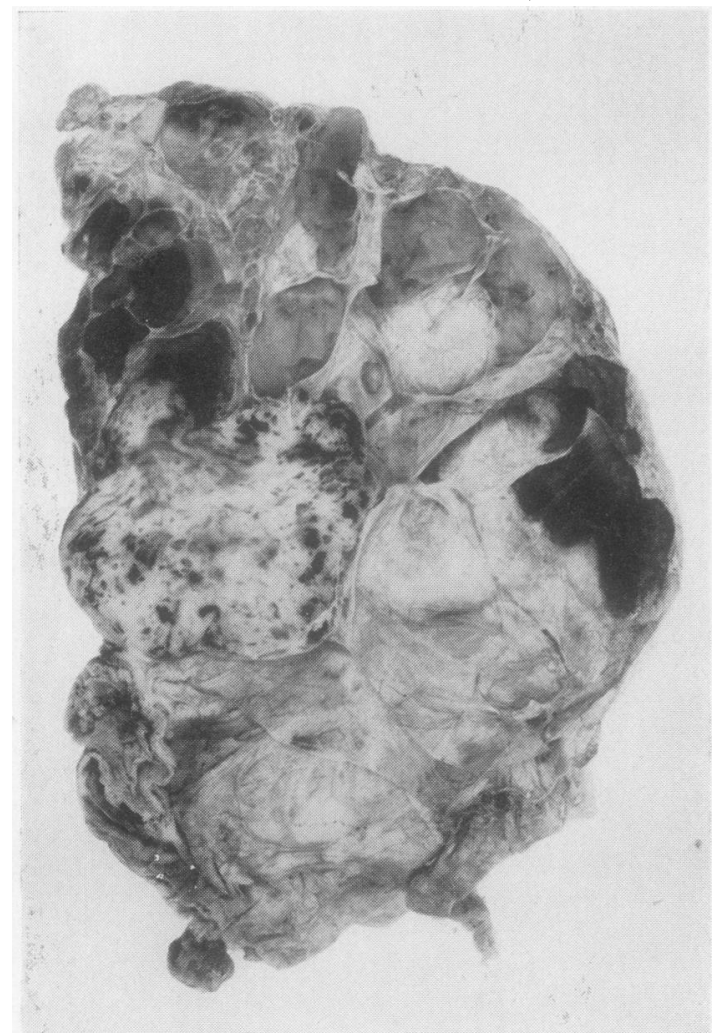

Fig. 7.-The thymic cyst removed from an 18-year-old boy whose radiographs are shown in Figs. 4 and 5. The solid element had the histological features of a benign thymoma. suspended from the upper anterior mediastinum by a narrow neck joining two flabby, translucent sacs, the larger sac reaching to the right dome of the diaphragm, and the smaller encroaching on the left pleural space. Both cysts were enucleated after incision of the reflection of mediastinal pleura over the part that projected into the right pleural space and after ligation of a large vessel that originated from the aorta ; in the man the left pleural space was widely opened. In the cyst resected from the boy (Fig. 7) there was a considerable solid element; the solid part of this lesion was called a thymoma, probably benign, by the panel of pathologists, and because of the large cyst in relation to it the lesion was called a cystic thymoma. The histological features of the cyst resected from the man were similar, but in this instance the solid elements associated with the cyst were much less obvious, and resembled more normal thymus.

Larmi (1960) reported 19 thymic tumours, eight of which were cystic. Keech, Storey, and Umiker (1954) and Ringertz and Lidholm (1956) described cysts possibly of thymic origin, and Willis (1958) has reviewed the literature relating to cysts of thymic origin. These cysts may be lined by columnar or ciliated epithelium, or, when they develop from Hassall's corpuscles, by stratified squamous epithelium, and they may be multilocular. Multilocular cysts, not recognizably of thymic origin, lying in the anterior mediastinum, and of a shape similar to the last two cysts described above, have been reported (Gerbasi, 1954 ; le Roux, 1959).

\section{Benign Tumours of the Thymus}

Seven symptomless patients, five of them women, were submitted to thoracotomy because they were found on routine radiography to have an opacity in the anterior mediastinum. The radiographs of two of these patients are shown (Figs. 8-10). Preliminary investigations in these patients contributed nothing, and in all the provisional diagnosis was thymic tumour or mediastinal teratoma. The radiographs of the patient shown in Figs. 8 and 9 were reproduced in an unrelated patient of the same sex a year later. The first of these two patients was regarded as having a teratoma ; the second, on the basis of the histological features of the tumour resected from the other patient, was correctly forecast as having a thymoma. The position of the tumour in three of these seven patients was lower than that generally recognized as typical of thymic 


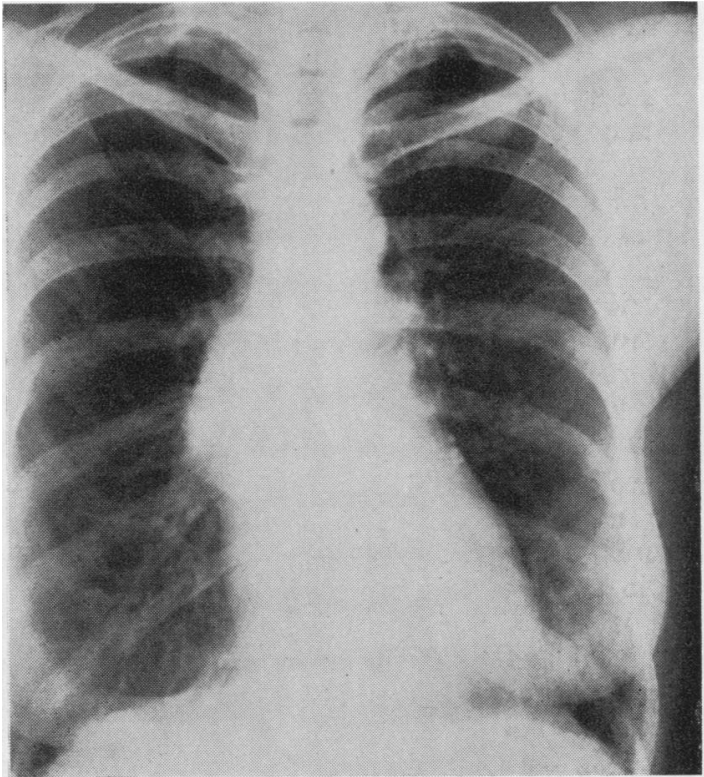

Fig. 8

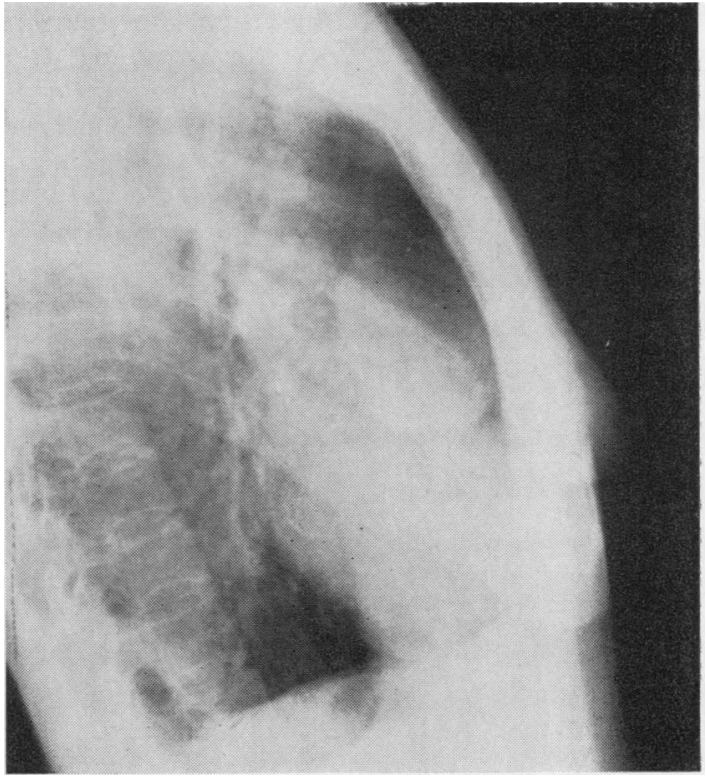

Fig. 9

Figs. 8 and 9.-This anterior mediastinal tumour lies low down on the pericardium, but was a benign thymic tumour.

tumours. Normal thymus extends on to the pericardium.

Three of these patients were in the fifth decade, three in the sixth decade, and the last was 70 years old. At thoracotomy, right-sided in three and leftsided in four, the tumours were all encapsulated,

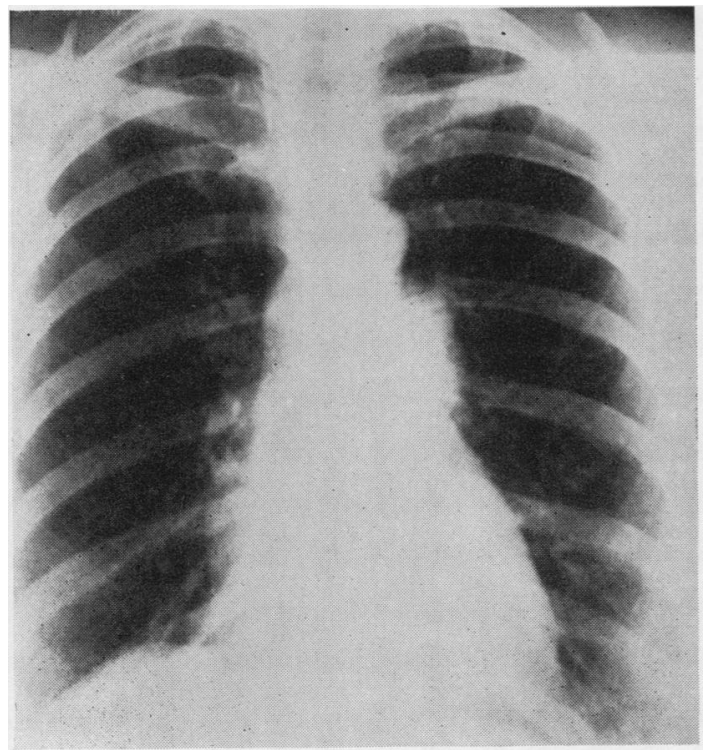

Fig. 10.-Another example of an anterior mediastinal tumour of thymic origin and surgically benign. in the anterior mediastinum, and in the position of the thymus; all were shelled cleanly from the mediastinum without the need for ligation of a pedicle; the patients recovered uneventfully from thoracotomy and all are well three to seven years after thoracotomy.

There is variation in the histological interpretation of these seven tumours, but all are regarded as of thymic origin, and are called tumours of mixed lympho-epithelial type by the majority of the panel of pathologists who examined them. One of the tumours is called benign by all who examined sections from it, and two pathologists called this an example of thymic hyperplasia. There is no unanimity of opinion regarding the histological benignity of the six remaining tumours; mitoses and capsular invasion are described by some pathologists and not by others, and these features are variously interpreted. In three of the tumours the lymphocytic elements appeared to preponderate ; in two, cystic spaces or clefts were described; in two others the lymphocytic and epithelial elements were so nearly normally represented that thymic hyperplasia was diagnosed by some, while others called the same lesions benign or malignant thymic tumours. Since many of the histologists examined different sections from the same tumour, these differences in interpretation may be the result of variation in the 
histological features in different parts of the same tumour. The justifications for grouping these seven patients together are the ease with which the tumours were shelled out of the mediastinum, the relative benignity of the tumours in the histological interpretation of most of the examining pathologists, and the well-being of the patients long after resection of the lesions. There are many references in the literature on thymic tumours to the difficulty of deciding whether a thymic tumour is benign or malignant on histological grounds, or whether it is indeed a tumour and not simply hyperplasia of the thymus (Fisher et al., 1952; Effler and McCormack, 1956 ; Larmi, 1960).

\section{Malignant Tumours Probably of THYMIC ORIGIN}

Six patients, four of them women, were submitted to thoracotomy each because of an anterior mediastinal lesion found radiographically. Two, a man and a woman, presented because of symptoms, and the remaining four because of the finding of an opacity on a routine radiograph. Four of these six patients were under 20 years: three women, one with symptoms, and a boy of 14 . One woman was 40 years of age; the oldest patient was a man of 65 who presented with symptoms. Preliminary investigations undertaken in the four patients without symptoms were unhelpful. In three the pre-operative diagnosis was thymic tumour or teratoma. Radiographs from two of these patients are shown (Figs. 11 and 12). In the fourth (Fig. 13) tomograms suggested that there might be two separate opacities in the anterior mediastinum, and in this patient the lesion was thought likely to be a reticulosis or sarcoid. Invasive tumours were found in all four patients. Three were approached from the left and one from the right. The tumours lay in the anterior mediastinum in the position of the thymus and in none was normal thymus recognized. The tumours were incompletely encapsulated, and in two there were associated enlarged glands close to the tumour. In one patient in whom a left thoracotomy was made, and in the patient submitted to right thoracotomy, the tumours were in two distinct parts. The lesion approached from the right invaded the anterior segment of the right upper lobe, and this segment was resected with the mediastinal mass. Part of this tumour was cystic; its surface was bossed and in parts ulcerated. These tumours had some or all of the macroscopic features associated with malignancyinvasion, ulceration, and dissemination.
The histological reports on the three tumours resected from the left include the terms lymphoma of the thymus, thymoma of low-grade malignancy, thymic lymphosarcoma, thymoma without further qualification, lymphosarcoma without further qualification, and Hodgkin's sarcoma. The tumour resected from the right was clinically the most clearly malignant, and this tumour was described histologically as a well-differentiated thymoma without mitotic activity by the only pathologist who examined it. These four patients were irradiated post-operatively. All are well two to 10 years after thoracotomy.

Two patients in this group of six had symptoms. The man aged 65 complained of vague nonpleuritic anterior chest pain for nine months. Radiographs of this patient are shown in Figs. 14 and 15. Preliminary routine investigations were unhelpful and thoracotomy was undertaken with the provisional diagnosis of anterior mediastinal teratoma. A spherical mass $8 \mathrm{~cm}$. in diameter was found in the anterior mediastinum, and after incision of the pleural reflection over this the tumour shelled cleanly from its bed, except in one part where the pleural covering was invaded, and here the surface of the tumour was ulcerated. Two enlarged glands lay close to the tumour and these were also resected. The tumour was called by four pathologists a malignant thymoma and by three a benign thymoma of mixed lymphoepithelial type. The glands were examined by another pathologist, who reported these as showing the changes of lymphosarcoma or lymphatic leukaemia. Because of the clinical evidences of malignancy-ulceration and adenopathy-the mediastinum of this patient was irradiated postoperatively. He died four months later with diffuse lymph-glandular enlargement and hepatomegaly: permission for a necropsy was not obtained. Death seems likely to have been a consequence of a diffuse malignant lymphoma or leukaemia.

The second patient with symptoms was a girl of 18. Two years earlier a chest radiograph was reported as normal. She presented with complaints of lack of well-being and loss of weight of short duration. A radiograph of this patient is shown in Fig. 16. She was recognized as having an anterior mediastinal tumour with a pleural and a pericardial effusion. Radioscopically the left dome of the diaphragm moved paradoxically. A sample of the pericardial effusion was shown to contain $4 \mathrm{~g}$. \% of protein and was not shown to contain malignant cells. Other investigations were unhelpful. At thoracotomy an 

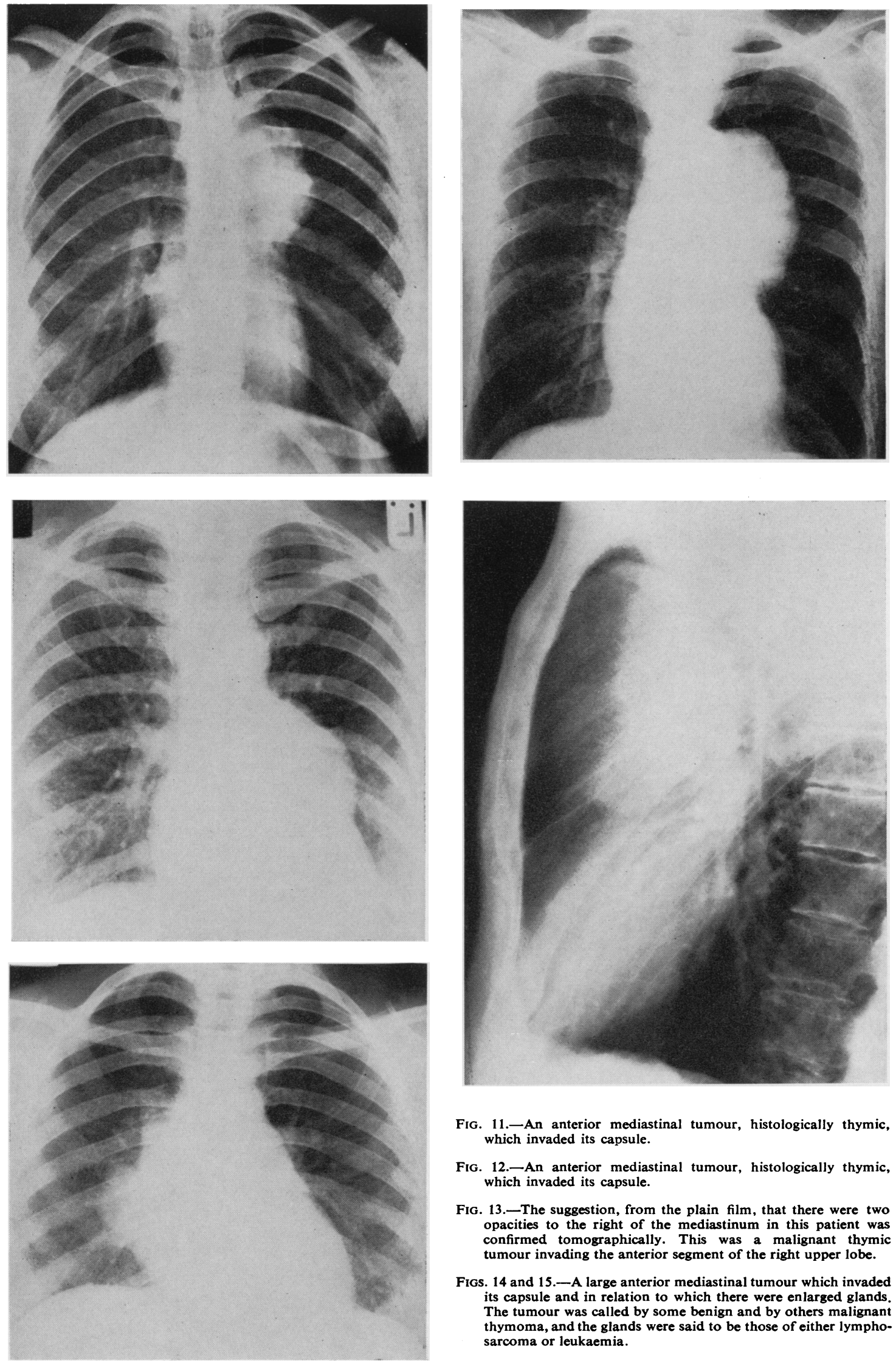

FIG. 11.-An anterior mediastinal tumour, histologically thymic, which invaded its capsule.

FIG. 12.-An anterior mediastinal tumour, histologically thymic, which invaded its capsule.

FIG. 13.-The suggestion, from the plain film, that there were two opacities to the right of the mediastinum in this patient was confirmed tomographically. This was a malignant thymic tumour invading the anterior segment of the right upper lobe.

Figs. 14 and 15.-A large anterior mediastinal tumour which invaded its capsule and in relation to which there were enlarged glands. The tumour was called by some benign and by others malignant thymoma, and the glands were said to be those of either lymphosarcoma or leukaemia. 


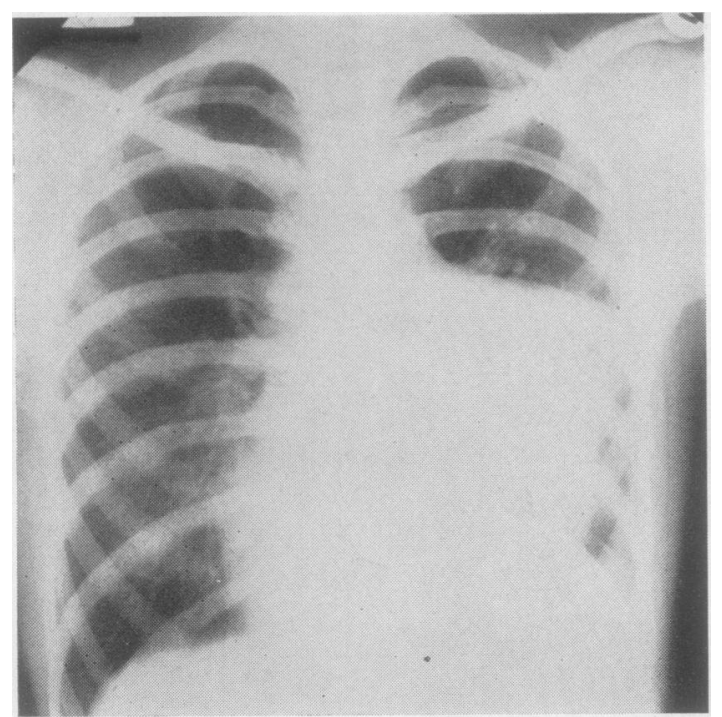

FIG. 16.-This anterior mediastinal tumour was recognized pre-operatively as producing a pericardial and a left pleural effusion and a left phrenic paresis.

invasive anterior mediastinal tumour was resected together with a large part of the pericardium, on the inner surface of which the tumour ulcerated, and with the upper lobe of the left lung which was widely invaded. Part of the left innominate vein, which was the cephalad limit of the tumour, was also resected because of invasion. The girl was undisturbed by thoracotomy and by the partial venous obstruction evident in the left side of the neck from the time of operation. The histological features of the tumour were those of sclerosing Hodgkin's disease, and the examining pathologists stated that the disease had arisen in the thymus. The girl remains well 18 months after thoracotomy and 16 months after completing a course of radiotherapy. Thoracotomy had been undertaken primarily to establish a diagnosis. It had been recognized pre-operatively that the tumour was likely to be malignant, but she might have had an infected teratomatous cyst to account both for the effusions and the phrenic paralysis.

\section{Anterior Mediastinal Tumours with Clinical EVIDENCE OF DisSEMINATION}

Nineteen patients were investigated because of a variety of symptoms and were found to have an anterior mediastinal tumour with evidence of dissemination. In nine of these patients the anterior mediastinal lesion was a well-defined opacity and the evidence of dissemination was cervical adenopathy. In 10 patients the anterior mediastinal lesion was ill defined, the mediastinum was broad, and these 10 patients all had the clinical features of obstruction of the superior vena cava. Four of these 10 had in addition cervical lymphadenopathy.

In those nine patients, eight of whom were women, who were found to have a localized anterior mediastinal opacity and enlarged cervical glands, the symptoms with which they presented were vague, and included tiredness, loss of weight, and ill-defined discomfort in the chest. A radiograph from one of these patients is shown (Fig. 17). The youngest patient was 14 and the oldest 69 ; most were over 40 years of age. A histological diagnosis was achieved in all nine patients by the examination of a resected neck gland. In none was thoracotomy undertaken. Blood findings in all were normal. In none was there evidence of dissemination other than to neck glands at the time when the patients were first seen. The interpretation of the histological features of the neck glands examined differed in different hands, but the consensus of opinion was that in four patients the features were those of Hodgkin's disease, in four those of lymphosarcoma, and in one those of reticulum-cell sarcoma. All nine patients were irradiated, with reduction in the size of the mediastinal opacity and decrease in size of the cervical glands.

One of these patients, the youngest in this group, is alive and well six years after irradiation. There are at the present time no clinical abnormalities, and the small residual mediastinal opacity has not changed during the six years since irradiation. In this patient the neck gland was said to show the features of lymphosarcoma. Two other patients are alive four and two years respectively after irradiation, but both have, in the interim, required irradiation of axillary and groin glands and one has required irradiation because of back pain and vertebral destruction. All the others died three months to three years after first being investigated as a result of a diffuse malignant lymphoma. One died from a cerebral catastrophe which may have been vascular, but at the time of her death there was clinical evidence of disseminated lymphoma.

Ten patients in this group had superior caval obstruction when they first presented. Nine of these were men. In most of them symptoms were directly attributable to caval obstruction. In addition to a broad mediastinum (Fig. 18), two of them had radiographic and radioscopic evidence of a pleural effusion and of interruption of a phrenic nerve (Fig. 19). Two others had tracheal 


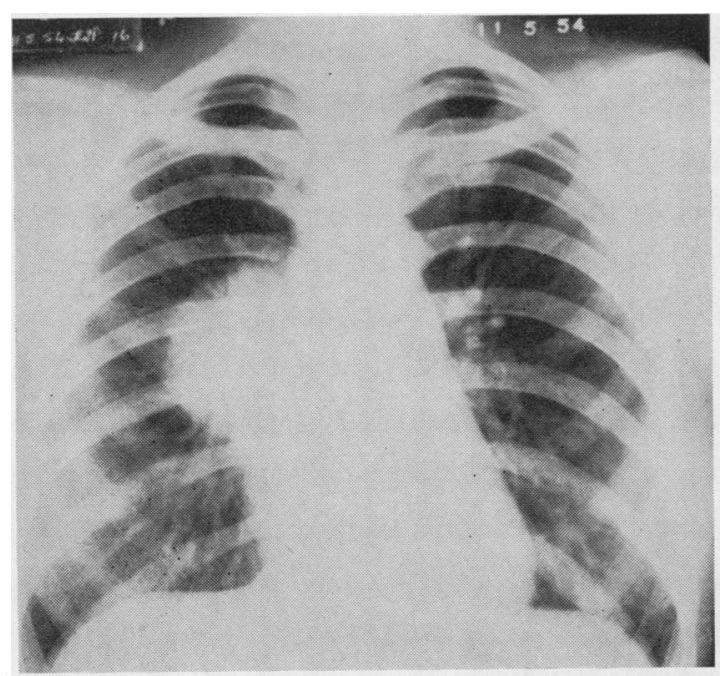

FIG. 17.-A well-defined opacity in the anterior mediastinum in a girl of 16 with cervical lymphadenopathy. The histological report on a cervical gland was lymphosarcoma.

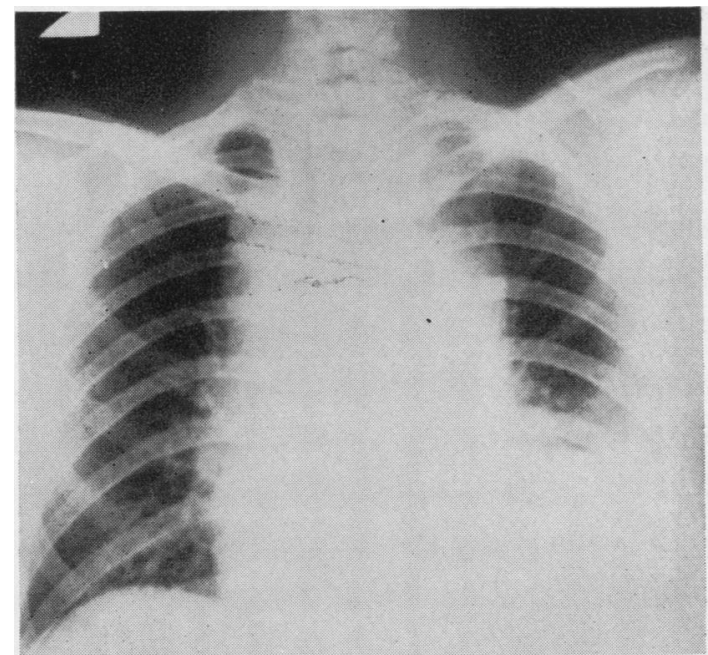

FIG. 19.-In this patient the broad mediastinum is associated with elevation of the left dome of the diaphragm and a left pleural effusion. He was irradiated in the absence of histological diagnosis. The lesion was ultimately shown to be a lymphosarcoma at necropsy.

compression producing stridor. In four there were enlarged neck glands, and in each a gland was removed for microscopy. In the two remaining patients abnormal tissue was seen bronchoscopically in one or other stem bronchus, and this tissue was examined histologically. Material for histo-

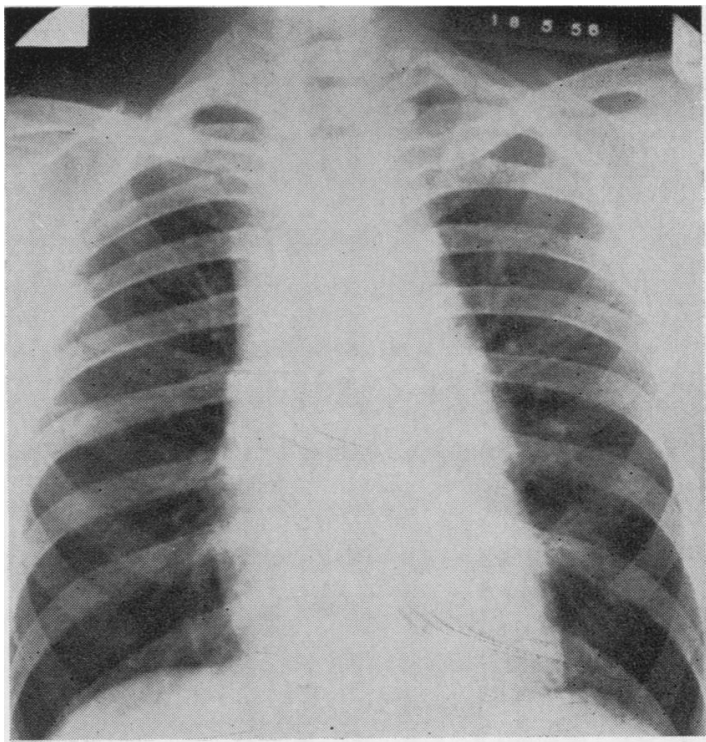

FIG. 18.-The broad mediastinum shown radiographically in this patient was associated with cervical lymphadenopathy interpreted histologically as lymphosarcoma.

logical examination was obtained in six of these 10 patients $-a$ neck gland from four and a bronchoscopic biopsy specimen from two. The histological features in all six were those of lymphosarcoma. No histological diagnosis was made in four at the time when they were first seen. All 10 patients were irradiated, the four without a histological diagnosis being regarded as having a malignant lymphoma. One of these four patients was alive six years later with a nearly normal radiograph and still lacking a histological diagnosis, but he cannot now be traced. The remaining nine patients died, some during radiotherapy and the remainder within a few months of irradiation. In the nine known to have died the necropsy findings were those of a diffuse malignant lymphoma, the predominating features being those of lymphosarcoma. All those who died were under 45 years of age and two were 12 years. If the remaining patient is alive he will at the present time be 43 . In the 10 patients with superior caval obstruction, just as in the nine patients with a well-defined anterior mediastinal lesion and cervical glandular enlargement, the only extrathoracic evidence of dissemination at the time of their first being seen was cervical adenopathy. While it is conceivable that the malignant lymphoma originated in neck glands, it is very much more likely that it originated in the mediastinum, possibly in the thymus. 


\section{Discussion}

The patients comprising this clinical appraisal of lesions possibly or certainly of thymic origin are few. Some features recur with noteworthy frequency. Women predominate, except in the group of malignant lymphomata with superior saval obstruction. The seven patients with macroscopically benign thymic tumours were middle-aged; none was young. The six patients with anterior mediastinal tumours regarded surgically as being malignant were mostly young ; four were under 20 years. The thymus is active in youth, and tumours in the young are notoriously invasive. Of nine patients with an anterior mediastinal opacity and cervical lymphadenopathy, eight were women. Of 10 patients with superior caval obstruction and a malignant lymphoma, nine were men, all were under 45 , and all but one aied within a few months of first being investigated.

The range of thymic lesions in the seriesthymic heterotopia, thymic cysts, thymic hyperplasia, benign and malignant thymomata, and malignant lymphomata possibly of thymic origin-includes most of the recognized lesions of the thymus. The variation in histological interpretation of the same tumour is a feature of all other reported series of thymic lesions. The absence of thymic tumours in patients submitted to thymectomy for myasthenia gravis, and of myasthenia in patients with thymic tumours, is unusual. The incidence of lesions certainly of thymic origin in comparison with other cysts and tumours in the mediastinum encountered in the same surgical unit (le Roux, $1960 \mathrm{a}, \mathrm{b})$ is $15 \%$. There is variation in the incidence of thymic lesions amongst other reported series of mediastinal tumours. Hodge, Aponte, and McLaughlin (1959) reported an incidence of $43 \%$. Ringertz and Lidholm (1956) and Larmi (1960) reported an incidence of $12 \%$. The incidence of thymic lesions amongst 241 mediastinal tumours collected from six series was $7.5 \%$ (Blades, 1946; Bradford, Mahon, and Grow, 1947 ; Brewer and Dolley, 1949 ; Curreri and Gale, 1949 ; Shidler and Holman, 1952).

\section{Summary}

Two examples of heterotopic thymus, four of thymic cysts, 13 of benign and malignant thymomata, and 19 of malignant anterior mediastinal lymphomata possibly of thymic origin are described. The lack of unanimity in the histological interpretation of thymomata justifies reliance on clinical and surgical experience rather than on a purely pathological classification of such tumours. The decision whether or not to submit a patient to radiotherapy after the removal of an anterior mediastinal tumour shown histologically to be of thymic origin has been made on the macroscopic features of ulceration, invasion, and dissemination and not on pathological grounds alone. In these patients no correlation has been found between thymic tumours and myasthenia gravis.

REFERENCES
Alter, N. M., and Osnato, M. (1930). Arch. Neurol. Psychiat. (Chicago), 23, 345.

Andritsakis, G D and Sommers, S. C. (1959). J.thorac. Surg., 37, 273. Blades, B. (1941). Amer. J. Surg., 54, 139.

(1946). Ann. Surg., 123, 749.

Bradford, M. L., Mahon, H. W., and Grow, J. B. (1947). Surg. Gynec. Obstet., 85, 467.

Brewer, L. A., and Dolley, F. S. (1949). Amer. Rev. Tuberc., 60, 419.

Clagett, O. T., and Eaton, L. M. (1943). Surg. Clin. N. Amer., 23, 1076 .

Cooper, T. V. (1950). J. clin. Path., 3, 48.

Curreri, A. R., and Gale, J. W. (1949). Arch. Surg. (Chicago), 58, 797.

Eaton, L. M., Clagett, O. T., Good, C. A., and McDonald, J. R. (1949). Arch. Neurol. Psychiat.'(Chicago), 61, 467.

Effler, D. B., and McCormack, L. J. (1956). J. thorac. Surg., 31, 60.

Fisher, E. R., Coburn, D., and Effler, D. B. (1952). Ibid., 24, 58.

Gerbasi, F. S. (1954). Ann. intern. Med. 41, 828.

Gerbasi, F. S. (1954) Ann. intern. Med., 41,828 . 78,402 .

Hodge, J., Aponte, G., and McLaughlin, E. (1959). J. thorac. Surg. 37, 730 .

Keech, W. G., Storey, C. F., and Umiker, W. C. (1954). Ibid. $27,477$.

Keynes, G. (1946). Brit. J. Surg., 33, 201.

(1955). Ibid., 42, 449.

Larmi, T. K. I. (1960). Ann. Chir. Gynaec. Fenn., 49, Suppl. 93.

le Roux, B. T. (1959). Thorax, 14, 27. (1960a). Ibid., 15, 339.

(1960b). Ibid., 15, 333.

(1961). Ibid., 16, 192 .

McBurney, R. P. Clagett, O. T., and McDonald, J. R. (1951). Proc Mayo Clin., 26, 345.

Murray, N. A., and McDonald, J. R. (1945). Amer. J. clin. Path. $15,87$.

Reid, H., and Marcus, R. (1949). Brit.J. Surg., 36, 271.

Ringertz, N., and Lidholm, S. O. (1956). J. thorac. Surg., 31, 458. Seybold, W. D., McDonald, J. R., Clagett, O. T., and Good, C. A. (1950). Ibid., 20, 195.

Shidler, F. P., and Holman, E. F. (1952). Stanf. med. Bull., 10, 217

Simpson, J. A. (1960). Personal communication.

Simpson, J. A. (1960). Personal communication. Willis, R. A. (1948). Pathology of Tumours. Butterworth, London.
(1958). The Borderland of Embryology and Pathology. Butterworth, London. 